background (NOD-S2+/-) to test the role of ER Ca2 + loss during T1D development. Senescence associated $\beta$ galactosidase staining (SA- $\beta$ gal), expression of senescence markers (RT-qPCR), mitochondrial function (Seahorse, TMRM) and mitochondrial copy number (qPCR) were all measured in S2KO versus WT $\beta$ cells and are currently being measured in the NOD-S2+/- mouse model at 6,8 , 12,14 , and $16 \mathrm{wks}$ of age. RESULTS/ANTICIPATED RESULTS: RT-qPCR assays detecting senescence markers cdkn1a and cdkn2a and mitochondrial specific genes cox 1 and nd1 were developed and validated in both INS-1 $\beta$ cells and mouse islets. Mitochondrial function assay (Seahorse) was optimized for use in INS- $1 \beta$ cells and is currently under development for use in intact mouse islets. S2KO $\beta$ cells displayed increased SA- $\beta$ gal staining as well as increased mitochondrial coupling efficiency $(\mathrm{p}=0.0146)$ and baseline mitochondrial copy number $(\mathrm{p}=0.0053)$ compared to WT $\beta$ cells, suggesting a senescence phenotype and altered mitochondrial function. NOD-S2+/- mice exhibited increased expression of the senescence marker cdkn2a in the islet at $12 \mathrm{wks}(\mathrm{p}=0.0117)$ compared to control mice, whereas cdknla remained unchanged across all timepoints tested. DISCUSSION/SIGNIFICANCE OF FINDINGS: Our results suggest that loss of SERCA2 and reduced ER Ca2 + alter $\beta$ cell mitochondrial function and are associated with features of senescence. Future studies will test whether SERCA2 activation and/or senolytic/senomorphic drugs are able to prevent or delay diabetes onset in NOD-S2+/- mice.

70759

\section{Jaw-specific control of Msx1-dependent odontogenesis by Dkk2 and Sostdc1}

Hyuk-Jae Edward Kwon

University at Buffalo

ABSTRACT IMPACT: Our proposed jaw-specific control mechanism of tooth development is expected to address the site-specific prevalence of tooth agenesis in humans. OBJECTIVES/GOALS: To determine the molecular mechanisms that control jaw-specific tooth development. To identify the molecular basis of the site-specific prevalence of humans tooth agenesis cases. METHODS/STUDY POPULATION: We used three different genetically engineered

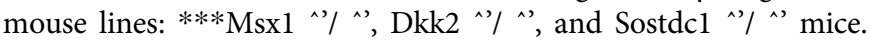
We used developmental mouse genetics approaches, basically generating different combinations of compound mutant mice. We examined their tooth development by using gross, histology, and mRNA expression analyses. RESULTS/ANTICIPATED RESULTS: We identified that Sostdc1, a secreted Wnt inhibitor, also plays an important role in regulating the Msx1-dependent odontogenic pathway. Sostdc1 mRNA showed similar expression patterns in the developing tooth germs between control and Msx1-null molar buds. Remarkably, by deleting the Sostdc1 gene, as well as the Dkk2 gene, in the Msx1-null background mouse, molar tooth development was rescued in the maxillary jaw, but not in the mandibular jaw. Furthermore, tooth developmental rescue could be achieved in both the maxillary and mandibular molars by combinedly deleting Dkk2 and Sostdc1 in Msx1-null mice. DISCUSSION/SIGNIFICANCE OF FINDINGS: Our study demonstrates that secreted Wnt inhibitors Dkk2 and Sostdc1 synergistically regulate the Msx1-dependent odontogenic pathway and further control early tooth morphogenesis. These mouse model will be used to further address the site-specific prevalence of tooth agenesis in humans.
Epigenetic Modification of Macrophages Contribute to Protective Memory in Against Staphylococcus aureus Liana C. Chan ${ }^{1}$, Mateo Pellegrini ${ }^{2}$, Colin Farrell ${ }^{2}$, Scott G. Filler ${ }^{1}$, Hong Lee ${ }^{3}$, Vance G. Fowler ${ }^{4}$, Jr., Elaine F. Reed ${ }^{2}$ and Michael R. Yeaman ${ }^{1}$

${ }^{1}$ Lundquist Institute for Biomedical Innovation at Harbor-UCLA, University of California, Los Angeles, CA, David Geffen SOM, ${ }^{2}$ University of California, Los Angeles, CA, ${ }^{3}$ Lundquist Institute for Biomedical Innovation at Harbor-UCLA and ${ }^{4}$ Duke University

ABSTRACT IMPACT: This work may provide new targets for vaccine and immunotherapeutic development against MRSA infections. OBJECTIVES/GOALS: Staphylococcus aureus is the leading cause of skin and skin structure infection (SSSI), a primary portal of entry for invasive infection. Patients with SA SSSI have a high 1-year recurrence. We have shown innate memory protects mice against SA SSSI. The goal of this project is to determine epigenetic mechanisms of protective memory against SA SSSI. METHODS/STUDY POPULATION: We have shown macrophages (Mf) afford protective memory against recurrent SA SSSI in mice. Priming by prior infection reduced skin lesion size and MRSA burden, which correlated with increased Mf in abscesses and lymph nodes. Priming potentiated the opsonophagocytic killing of SA by bone-marrow derived $\mathrm{Mf}(\mathrm{BMDM})$ in vitro, and their adoptive transfer into naive skin afforded protective efficacy in vivo. Here, we investigated epigenetic mechanisms of anti-SA efficacy in BMDMs. BMDM from naive (uninfected) or primed (SA SSSI) wild-type C57Bl/6 mice were cultured ex vivo. DNA from BMDM groups were isolated and analyzed for methylation changes using reduced representation bisulfite sequencing (RRBS). Pathway analyses of methylation changes were determined with Panther. RESULTS/ANTICIPATED RESULTS: Present findings indicate the protective memory afforded by BMDM was mediated by epigenetic modifications of the DNA. Using RRBS, we profiled differentially methylated regions (DMR) in DNA from naive vs. primed BMDM. Primed BMDM exhibited significantly different DMRs as compared to naive BMDM. Proximity to known genes were mapped using GREAT. Pathway analyses revealed DMRs predominant in genes integral to immune modulation, such as integrin signaling, cytokine/chemokine networks, and growth regulation. For example, SA-primed BMDM were hypermethylated proximate to GIMAP8 versus naive BMDM, suggesting repression of this protein. Gimap family ligands are small GTPase immune-associated proteins expressed in immune cells known to regulate macrophage lysosomal fusion during parasite infection. DISCUSSION/SIGNIFICANCE OF FINDINGS: These findings reveal epigenetic mechanisms of macrophage innate memory against recurrent MRSA infection. Functional testing of these genes in response to SA infection is needed to confirm their protective role. These insights may provide new targets for vaccine and immunotherapeutic development against MRSA.

79664

\section{Complement Driven Auto-Reactive Antibodies in Lung} Transplantation*

Alexander McQuiston ${ }^{1}$, Changhai $\mathrm{Li}^{1}$, Kunal Patel ${ }^{1}$, Zhenxiao Tu${ }^{1}$, Dianna Nord ${ }^{2}$, Satish Nadig ${ }^{1}$, Stephen Tomlinson ${ }^{1}$ and Carl Atkinson ${ }^{2}$ ${ }^{1}$ Medical University of South Carolina and ${ }^{2}$ University of Florida

ABSTRACT IMPACT: Our work unveils a novel mechanism of ischemia repurfusion injury driven by pre-existing autoimmunity following lung transplant and a potential therapeutic strategy for 